\title{
Crystal structure of $\operatorname{di}\left(\mu\right.$-benzoato- $\left.O, O^{\prime}\right) \operatorname{di}\left(\mu\right.$-benzoato- $\left.O, O^{\prime}: O^{\prime}\right)$ dinitratodi(2,2'-bipyridine)dipraseodymium(III), $\mathrm{Pr}_{2}\left(\mathrm{C}_{7} \mathrm{H}_{5} \mathrm{O}_{2}\right)_{4}\left(\mathrm{NO}_{3}\right)_{2}\left(\mathrm{C}_{10} \mathrm{H}_{8} \mathrm{~N}_{2}\right)_{2}$
}

\author{
S.-Y. Zhang and L.-G. Zhu* \\ Zhejiang University, Department of Chemistry, Hangzhou 310027, P. R. China
}

Received November 7, 2002, accepted and available on-line January 22, 2003; CCDC-No. 1267/969
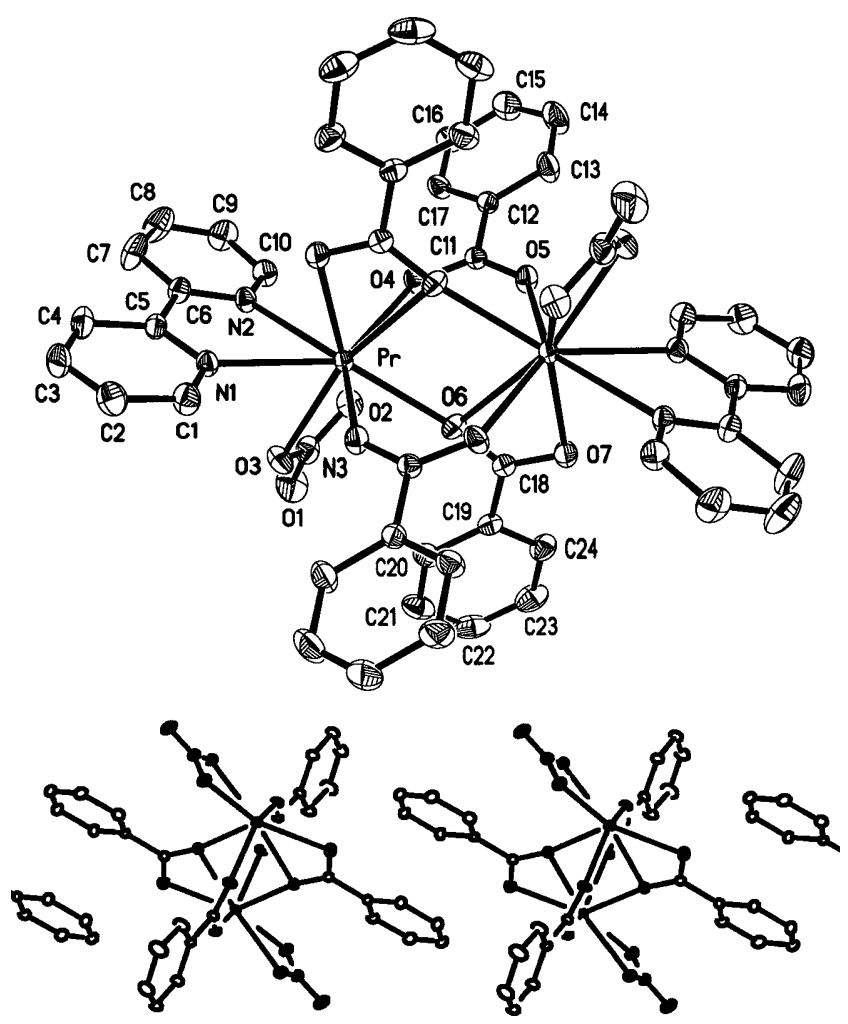

Abstract

$\mathrm{C}_{48} \mathrm{H}_{36} \mathrm{~N}_{6} \mathrm{O}_{14} \mathrm{Pr}_{2}$, triclinic, $P \overline{1}$ (No. 2), $a=10.2661(5) \AA$, $b=11.0301(5) \AA, c=11.0854(5) \AA, \alpha=97.723(1)^{\circ}$, $\beta=103.001(1)^{\circ}, \gamma=70.783(1)^{\circ}, V=1152.5 \AA^{3}, Z=1$, $R_{\mathrm{gt}}(F)=0.037, w R_{\mathrm{ref}}\left(F^{2}\right)=0.096, T=293 \mathrm{~K}$.

\section{Source of material}

The title complex was synthesized using mixed solution approach. $\mathrm{Pr}\left(\mathrm{NO}_{3}\right)_{3} \cdot 6 \mathrm{H}_{2} \mathrm{O}(0.2 \mathrm{mmol})$ was dissolved in a mixture of ethanol $(5 \mathrm{ml})$ and water $(2 \mathrm{ml})$. A solution of 2,2'-bipyridine (0.63 mmol), 8-hydroxyquinoline (HQ, $1.0 \mathrm{mmol}$ ) and benzoic acid (HBA, $9.83 \mathrm{mmol})$ in ethanol $(10 \mathrm{ml})$ was added to the above solution. After about one week, crystals were obtained. The 8-hydroxyquinoline is key factor to formation of quaternary complexes. If there is no HQ in the synthetic system we couldn't obtain any product because of the strong acidity. The HQ can decrease the acidity of the solution and form $\mathrm{H}_{2} \mathrm{Q}^{+}$with $\mathrm{H}^{+}$, which is difficult to bind $\mathrm{Ln}^{3+}$, and keep in the solution a certain amount of benzoate anions. The quantity of HBA should exceed $1.0 \mathrm{~g}$

\footnotetext{
* Correspondence author (e-mail: chezlg@zju.edu.cn)
}

$(8.19 \mathrm{mmol})$ so that clear solution could be obtained and after one week nice crystals were formed. Otherwise, yellow complex containing HQ ligand was precipitated and IR showed no presence of benzoate ligand. In the case of the yellow precipitate while the amount of HBA exceeded $0.6 \mathrm{~g}$ (4.91 mmol), the yellow complex was gradually changed into green complex (the title complex), which indicated ligand exchange between $\mathrm{Q}^{-}$and $\mathrm{BA}^{-}$. Elemental analysis for $\mathrm{C}_{48} \mathrm{H}_{36} \mathrm{~N}_{6} \mathrm{O}_{14} \mathrm{Pr}_{2}$ : (calculated) $\mathrm{C}, 47.94 \%$; N, 6.99\%; $\mathrm{H}, 3.02 \%$; Pr, 23.43\%; (found) C, 47.73\%; N, 6.83\%; H, 3.04\%; $\operatorname{Pr}, 23.27 \%$.

\section{Discussion}

The first crystal structure of quaternary complexes of lanthanide with two anionic ions was reported in our group [1] due to new synthetic procedure used. Then about 20 crystal structures were reported in our laboratory $[2,3]$ and other research groups $[4,5]$. These quaternary complexes have been extensively received many interests because of their special structures and interesting functional properties while most of complexes have the neutral ligand of 1,10-phenanthroline. To date, the title complex represents an exception, as only few complexes with $2,2^{\prime}$-bipyridine ligand have been studied.

The coordination geometry of the Pr atom can be described as a monocapped square antiprism with nine-coordinate. The complex is a dimer with a Pr-Pr separation of $4.004 \AA$ and possesses a center of symmetry. The coordination geometry involves five oxygen atoms from four benzoate anions, two nitrogen atoms from 2,2'-bipyridine and another two oxygen atoms from nitrate group. The four benzoate anions in the dimer complex show two coordination modes, which is very difficult to distinguish them from IR spectrum: one mode bonds to two Pr atoms through O4 and O5; the other mode is chelated to one Pr atom with distances of 2.427(3) $\AA$ and 2.716(3) $\AA$ for O6-Pr and O6-Pr*, respectively. The distance $\mathrm{Pr}-\mathrm{O}\left(\mathrm{NO}_{3}\right)$ is longer than $\mathrm{Pr}-\mathrm{O}(\mathrm{BA})$ except $\mathrm{Pr}-\mathrm{O6}^{*}$, attributed to strong bonding of benzoate with metal ion. The bipy ligands are situated in the vacant sides, and therefore the complex has little steric exclusion. The bond length of N3-O1 from the nitrate anion is 1.215(5) A, clearly double bond. The N3-O2 and N3-O3 bond lengths are 1.250(5) $\AA$ and 1.277(5) $\AA$, respectively, which are between single and double bonds. The nitrate anions can be replaced by other anions, such as chloride, acetate, etc.

The distance between $\pi-\pi$ stacked benzoate ligands from neighboring molecules is about $3.6 \AA$. Then, an extended structure (bottom figure), one-dimensional network, is formed, which is unavailable in the case of aliphatic acid and this stacking effect is more popuplar in quaternary complexes than that of ternary complexes. 
Table 1. Data collection and handling.

\begin{tabular}{ll}
\hline Crystal: & light green prism, size $0.2 \times 0.3 \times 0.5 \mathrm{~mm}$ \\
Wavelength: & Mo $K_{\alpha}$ radiation $(0.71073 \AA)$ \\
$\mu:$ & $21.65 \mathrm{~cm}^{-1}$ \\
Diffractometer, scan mode: & Bruker SMART CCD, $\varphi / \omega$ \\
$2 \theta_{\text {max }}:$ & $56.52^{\circ}$ \\
$N(h k l)_{\text {measured }}, N(h k l)_{\text {unique }}:$ & 7096,5141 \\
Criterion for $I_{\text {obs }}, N(h k l)_{\text {gt }}:$ & $I_{\text {obs }}>2 \sigma\left(I_{\text {obs }}\right), 4990$ \\
$N(\text { param })_{\text {refined }}$ & 389 \\
Program: & SHELXTL [6] \\
& \\
\hline
\end{tabular}

Table 2. Atomic coordinates and displacement parameters (in $\AA^{2}$ ).

\begin{tabular}{llllll}
\hline Atom & Site & $x$ & $y$ & $z$ & $U_{\text {iso }}$ \\
\hline $\mathrm{H}(1)$ & $2 i$ & $0.974(6)$ & $-0.104(5)$ & $0.590(5)$ & $0.06(1)$ \\
$\mathrm{H}(2)$ & $2 i$ & $1.196(6)$ & $-0.080(5)$ & $0.623(5)$ & $0.06(2)$ \\
$\mathrm{H}(3)$ & $2 i$ & $1.272(7)$ & $0.028(6)$ & $0.808(6)$ & $0.08(2)$ \\
$\mathrm{H}(4)$ & $2 i$ & $1.104(5)$ & $0.150(4)$ & $0.940(4)$ & $0.04(1)$ \\
$\mathrm{H}(5)$ & $2 i$ & $0.939(5)$ & $0.255(7)$ & $1.030(7)$ & $0.10(2)$ \\
$\mathrm{H}(6)$ & $2 i$ & $0.77(1)$ & $0.340(9)$ & $1.163(9)$ & $0.12(3)$ \\
$\mathrm{H}(7)$ & $2 i$ & $0.561(6)$ & $0.293(5)$ & $1.086(5)$ & $0.05(1)$ \\
$\mathrm{H}(8)$ & $2 i$ & $0.532(3)$ & $0.155(5)$ & $0.924(5)$ & $0.04(1)$ \\
$\mathrm{H}(9)$ & $2 i$ & $0.033(6)$ & $0.328(5)$ & $0.511(2)$ & $0.05(2)$ \\
$\mathrm{H}(10)$ & $2 i$ & $-0.13(1)$ & $0.49(1)$ & $0.58(1)$ & $0.16(5)$ \\
$\mathrm{H}(11)$ & $2 i$ & $-0.068(8)$ & $0.562(7)$ & $0.824(7)$ & $0.09(2)$ \\
$\mathrm{H}(12)$ & $2 i$ & $0.131(8)$ & $0.470(6)$ & $0.922(3)$ & $0.07(2)$ \\
$\mathrm{H}(13)$ & $2 i$ & $0.303(8)$ & $0.306(7)$ & $0.838(7)$ & $0.08(2)$ \\
$\mathrm{H}(14)$ & $2 i$ & $0.655(6)$ & $-0.337(6)$ & $0.664(6)$ & $0.07(2)$ \\
$\mathrm{H}(15)$ & $2 i$ & $0.680(4)$ & $-0.505(8)$ & $0.779(7)$ & $0.11(3)$ \\
$\mathrm{H}(16)$ & $2 i$ & $0.497(6)$ & $-0.597(6)$ & $0.763(6)$ & $0.07(2)$ \\
$\mathrm{H}(17)$ & $2 i$ & $0.286(5)$ & $-0.507(6)$ & $0.638(6)$ & $0.07(2)$ \\
$\mathrm{H}(18)$ & $2 i$ & $0.248(3)$ & $-0.335(5)$ & $0.530(5)$ & $0.07(2)$ \\
\hline
\end{tabular}

Table 3. Atomic coordinates and displacement parameters (in $\AA^{2}$ ).

\begin{tabular}{|c|c|c|c|c|c|c|c|c|c|c|}
\hline Atom & Site & $x$ & $y$ & $z$ & $U_{11}$ & $U_{22}$ & $U_{33}$ & $U_{12}$ & $U_{13}$ & $U_{23}$ \\
\hline $\operatorname{Pr}$ & $2 i$ & $0.64028(2)$ & $0.00029(1)$ & $0.65798(1)$ & $0.0221(1)$ & $0.0300(1)$ & $0.0225(1)$ & $-0.00640(7)$ & $0.00275(7)$ & $0.00123(7)$ \\
\hline $\mathrm{N}(1)$ & $2 i$ & $0.9032(3)$ & $0.0071(3)$ & $0.7292(3)$ & $0.025(1)$ & $0.040(2)$ & $0.037(2)$ & $-0.009(1)$ & $0.006(1)$ & $0.000(1)$ \\
\hline $\mathrm{N}(2)$ & $2 i$ & $0.7104(3)$ & $0.1242(3)$ & $0.8711(3)$ & $0.031(1)$ & $0.043(2)$ & $0.028(1)$ & $-0.010(1)$ & $0.003(1)$ & $-0.002(1)$ \\
\hline $\mathrm{N}(3)$ & $2 i$ & $0.6767(4)$ & $-0.1662(4)$ & $0.8622(3)$ & $0.051(2)$ & $0.053(2)$ & $0.033(2)$ & $-0.023(2)$ & $-0.001(1)$ & $0.010(1)$ \\
\hline $\mathrm{O}(1)$ & $2 i$ & $0.6965(5)$ & $-0.2345(4)$ & $0.9461(3)$ & $0.090(3)$ & $0.093(3)$ & $0.050(2)$ & $-0.037(2)$ & $-0.005(2)$ & $0.040(2)$ \\
\hline $\mathrm{O}(2)$ & $2 i$ & $0.5621(3)$ & $-0.0815(3)$ & $0.8298(3)$ & $0.042(2)$ & $0.058(2)$ & $0.041(1)$ & $-0.015(1)$ & $0.013(1)$ & $0.008(1)$ \\
\hline $\mathrm{O}(3)$ & $2 i$ & $0.7743(3)$ & $-0.1779(3)$ & $0.8034(3)$ & $0.037(1)$ & $0.052(2)$ & $0.053(2)$ & $-0.005(1)$ & $0.003(1)$ & $0.019(1)$ \\
\hline $\mathrm{O}(4)$ & $2 i$ & 0.4093(3) & $0.1432(3)$ & $0.6766(2)$ & $0.027(1)$ & $0.045(1)$ & $0.034(1)$ & $0.000(1)$ & $0.0060(9)$ & 0.001(1) \\
\hline $\mathrm{O}(5)$ & $2 i$ & $0.2496(3)$ & $0.1366(3)$ & $0.5048(2)$ & $0.032(1)$ & $0.049(1)$ & $0.032(1)$ & $-0.009(1)$ & $0.007(1)$ & $-0.010(1)$ \\
\hline $\mathrm{O}(6)$ & $2 i$ & $0.4921(3)$ & $-0.1281(2)$ & $0.5505(2)$ & $0.043(1)$ & $0.037(1)$ & $0.036(1)$ & $-0.022(1)$ & $0.008(1)$ & $-0.001(1)$ \\
\hline $\mathrm{O}(7)$ & $2 i$ & $0.3285(3)$ & $-0.1874(3)$ & $0.4187(3)$ & $0.044(2)$ & $0.043(1)$ & $0.052(2)$ & $-0.020(1)$ & $-0.009(1)$ & $0.015(1)$ \\
\hline $\mathrm{C}(1)$ & $2 i$ & $0.9986(4)$ & $-0.0527(5)$ & $0.6587(4)$ & $0.037(2)$ & $0.058(2)$ & $0.049(2)$ & $-0.014(2)$ & $0.015(2)$ & $-0.009(2)$ \\
\hline $\mathrm{C}(2)$ & $2 i$ & $1.1346(5)$ & $-0.0455(5)$ & $0.6852(5)$ & $0.036(2)$ & $0.063(3)$ & $0.071(3)$ & $-0.013(2)$ & $0.023(2)$ & $0.001(2)$ \\
\hline $\mathrm{C}(3)$ & $2 i$ & $1.1752(4)$ & $0.0235(5)$ & $0.7895(5)$ & $0.032(2)$ & $0.064(3)$ & $0.071(3)$ & $-0.022(2)$ & $0.008(2)$ & $0.007(2)$ \\
\hline $\mathrm{C}(4)$ & $2 i$ & $1.0775(5)$ & $0.0894(5)$ & $0.8626(5)$ & $0.038(2)$ & $0.063(3)$ & $0.053(2)$ & $-0.025(2)$ & $-0.001(2)$ & $0.005(2)$ \\
\hline $\mathrm{C}(5)$ & $2 i$ & $0.9425(4)$ & $0.0779(3)$ & $0.8301(3)$ & $0.032(2)$ & $0.039(2)$ & $0.035(2)$ & $-0.015(1)$ & $0.002(1)$ & $0.004(1)$ \\
\hline $\mathrm{C}(6)$ & $2 i$ & $0.8354(4)$ & $0.1455(4)$ & $0.9061(3)$ & $0.039(2)$ & $0.043(2)$ & $0.032(2)$ & $-0.017(2)$ & $0.004(1)$ & $-0.002(1)$ \\
\hline $\mathrm{C}(7)$ & $2 i$ & $0.8620(6)$ & $0.2248(6)$ & $1.0118(5)$ & $0.067(3)$ & $0.087(4)$ & $0.051(3)$ & $-0.050(3)$ & $0.016(2)$ & $-0.027(2)$ \\
\hline $\mathrm{C}(8)$ & $2 i$ & $0.7601(7)$ & $0.2812(6)$ & $1.0812(5)$ & $0.089(4)$ & $0.082(4)$ & $0.057(3)$ & $-0.048(3)$ & $0.028(3)$ & $-0.036(3)$ \\
\hline $\mathrm{C}(9)$ & $2 i$ & $0.6332(6)$ & $0.2583(5)$ & $1.0464(4)$ & $0.063(3)$ & $0.063(3)$ & $0.046(2)$ & $-0.019(2)$ & $0.023(2)$ & $-0.018(2)$ \\
\hline $\mathrm{C}(10)$ & $2 i$ & $0.6132(4)$ & $0.1774(4)$ & $0.9409(4)$ & $0.038(2)$ & $0.049(2)$ & $0.038(2)$ & $-0.009(2)$ & $0.011(2)$ & $-0.006(2)$ \\
\hline $\mathrm{C}(11)$ & $2 i$ & $0.2878(4)$ & $0.1827(3)$ & $0.6107(3)$ & $0.028(2)$ & $0.032(2)$ & $0.031(2)$ & $-0.007(1)$ & $0.009(1)$ & $0.000(1)$ \\
\hline $\mathrm{C}(12)$ & $2 i$ & $0.1823(3)$ & $0.2919(3)$ & $0.6668(3)$ & $0.029(2)$ & $0.033(2)$ & $0.034(2)$ & $-0.007(1)$ & $0.008(1)$ & $-0.001(1)$ \\
\hline $\mathrm{C}(13)$ & $2 i$ & $0.0537(5)$ & $0.3540(5)$ & $0.5946(5)$ & $0.037(2)$ & $0.059(3)$ & $0.050(2)$ & $0.003(2)$ & $-0.004(2)$ & $-0.013(2)$ \\
\hline $\mathrm{C}(14)$ & $2 i$ & $-0.0407(6)$ & $0.4566(6)$ & $0.6473(6)$ & $0.039(2)$ & $0.070(3)$ & $0.079(4)$ & $0.015(2)$ & $-0.003(2)$ & $-0.014(3)$ \\
\hline$C(15)$ & $2 i$ & $-0.0107(5)$ & $0.4949(5)$ & $0.7703(6)$ & $0.047(2)$ & $0.054(3)$ & $0.074(3)$ & $0.005(2)$ & $0.016(2)$ & $-0.018(2)$ \\
\hline $\mathrm{C}(16)$ & $2 i$ & $0.1148(5)$ & $0.4341(4)$ & $0.8423(4)$ & $0.055(2)$ & $0.044(2)$ & $0.043(2)$ & $-0.003(2)$ & $0.016(2)$ & $-0.009(2)$ \\
\hline $\mathrm{C}(17)$ & $2 i$ & $0.2122(4)$ & $0.3333(4)$ & $0.7896(4)$ & $0.038(2)$ & $0.040(2)$ & $0.035(2)$ & $-0.001(2)$ & $0.006(2)$ & $0.001(1)$ \\
\hline $\mathrm{C}(18)$ & $2 i$ & $0.4206(4)$ & $-0.2047(3)$ & $0.5146(3)$ & $0.033(2)$ & $0.032(2)$ & $0.032(2)$ & $-0.010(1)$ & $0.010(1)$ & $0.000(1)$ \\
\hline $\mathrm{C}(19)$ & $2 i$ & $0.4470(4)$ & $-0.3176(3)$ & $0.5876(3)$ & $0.042(2)$ & $0.030(2)$ & $0.033(2)$ & $-0.012(1)$ & $0.011(1)$ & $-0.001(1)$ \\
\hline $\mathrm{C}(20)$ & $2 i$ & $0.5739(5)$ & $-0.3701(4)$ & $0.6644(4)$ & $0.046(2)$ & $0.039(2)$ & $0.043(2)$ & $-0.005(2)$ & $0.007(2)$ & $0.003(2)$ \\
\hline $\mathrm{C}(21)$ & $2 i$ & $0.5932(6)$ & $-0.4755(5)$ & $0.7299(5)$ & $0.070(3)$ & $0.044(2)$ & $0.048(2)$ & $0.000(2)$ & $0.011(2)$ & $0.014(2)$ \\
\hline $\mathrm{C}(22)$ & $2 i$ & $0.4851(7)$ & $-0.5270(4)$ & $0.7181(5)$ & $0.111(5)$ & $0.036(2)$ & $0.050(3)$ & $-0.017(2)$ & $0.031(3)$ & $0.008(2)$ \\
\hline $\mathrm{C}(23)$ & $2 i$ & $0.3594(7)$ & $-0.4755(5)$ & $0.6422(5)$ & $0.088(4)$ & $0.050(3)$ & $0.060(3)$ & $-0.038(3)$ & $0.023(3)$ & $0.003(2)$ \\
\hline $\mathrm{C}(24)$ & $2 i$ & $0.3391(5)$ & $-0.3713(4)$ & $0.5749(4)$ & $0.056(2)$ & $0.045(2)$ & $0.049(2)$ & $-0.027(2)$ & $0.009(2)$ & $0.005(2)$ \\
\hline
\end{tabular}

\section{References}

1. Dong, N.; Zhu, L. G.; Xu, C.: Synthesis and crystal structure of the lanthanide complex with mixed ligands. Chinese J. Struct. Chem. 12 (1993) 133-136.

2. Zhu, L. G.; Xie, X. P.; Yu, Q. S.: Synthesis and crystal structure of quaternary mixed anion complexes of lanthanide containing 1,10-phenanthroline. J. Rare Earths 17 (1999) 12-15.

3. Zhu, L. G.; Kitagawa, S.: Crystal structure, stacking effect and 2D network assembled by hydrogen bonds of quaternary complex. Chem. J. Chin. Universities 22 (2001) 188-190.
4. Niu, S. Y.; Yang, Z. Z.; Yang, Q. C.; Yang, B.; Chao, J. Q.; Yang, G. D.; Shen, E. Z.: Structure and magnetism of tetraacetato bridged binuclear Nd(III) complex. Polyhedron 16 (1997) 1629-1635.

5. Li, X.; Jin, L. P.; Zheng, X. J.; Lu, S. Z.; Zhang, J. H.: Synthesis, structure and luminescence property of the three ternary and quaternary europium complexes. J. Mol. Struct. 607 (2002) 59-67.

6. Bruker, SHELXTL, Version 5.10. Bruker AXS, Madison, Wisconsin, USA 1998. 